\title{
Constructos y variables del ambiente virtual de aprendizaje, desde la perspectiva del modelo de ecuaciones estructurales
}

\section{Constructs and Variables of the Virtual Learning Environment, from the Perspective of Structural Equation Model}

\author{
Alfredo Ángel Ramírez-Carbajal \\ Universidad Autónoma del Estado de México. México. \\ posgradoarc@hotmail.com
}

\begin{abstract}
Resumen
Este artículo presenta el diseño y los resultados de un proyecto de investigación. Se inicia con la contextualización en el área educativa y enseguida se describe una aproximación a la revisión de la literatura sobre el ambiente virtual de aprendizaje y el modelo de ecuaciones estructurales. En la fundamentación, se explican las teorías de la acción razonada, la del modelo de aceptación de la tecnología, la del modelo de éxito de los sistemas de información y la del modelo de ecuaciones estructurales. Mediante el método, se logra recopilar 1175 artículos de distintas revistas científicas. A partir de estos y con la aplicación de una serie de criterios se identifica una muestra representativa de 421 . Los resultados dan cuenta de los constructos, las variables y el tipo de teorías aplicadas en los ambientes virtuales investigados. Por último, la discusión y las conclusiones están referidas a las asociaciones entre los elementos de los modelos publicados por sus autores.
\end{abstract}

\section{Palabras clave}

Artículo, educación a distancia, modelo, teoría, variable.

\begin{abstract}
This paper presents the design and results of a research project. It starts with the contextualization in education and then an approach to the review of the literature on the virtual learning environment and structural equation model is described. In the reasoning, theories of reasoned action, the model of acceptance of the technology, the successful model of information systems and structural equation model are explained. By the method it is achieved collect 1175 items of various scientific journals. From these and the implementation of a number of criteria a representative sample of 421 . The results show constructs, variables and type of theories applied in virtual environments investigated is identified. Finally, the discussion and conclusions are referred to associations between the elements of the models published by their authors.
\end{abstract}

\section{Key words}

Paper, distance education, model, theory, variable

\section{Introducción}

El incremento de las investigaciones de la educación a distancia (en forma cuantitativa y cualitativa) ha dado lugar a diversas áreas de estudio, tales como: formación basada en la tecnología de internet, aprendizaje distribuido, instrucción basada en la Web, aprendizaje en línea, aprendizaje flexible, ambiente virtual de aprendizaje, por 
mencionar a las más relevantes (existiendo en ciertos casos, algunas controversias por compartir determinadas características, entre estas). Con base a dichas áreas, se seleccionó al ambiente virtual de aprendizaje para realizar la investigación, considerando que el área elegida tiene como base común a las TIC. De igual forma, en la revisión de la literatura educativa el concepto de aprendizaje virtual tiene sus variantes, por un lado, las distintas opciones de poder usar una determinada tecnología, como internet, intranet o extranet y por otro, la forma de implementar un sinnúmero de aplicaciones de cómputo (entre otros). Por otra parte, Con el aumento de las necesidades de tecnología de igual forma se incrementó la cantidad de fallas en la adopción de un determinado sistema en las organizaciones, Chuttur (2009). La afirmación anterior, refleja el estado actual en donde el estudio de los sistemas se ha convertido en un área de interés para muchos investigadores. De acuerdo con lo anterior, se han propuesto algunas teorías a lo largo de las últimas cuatro décadas para explicar (o predecir) el uso de un sistema. Por estos motivos, el usuario y la aceptación de la tecnología, ha sido un importante campo de estudio desde la perspectiva de la teoría de sistemas. En consideración a los aspectos de pertinencia, significación, factibilidad y viabilidad se establecen las bases para la definición del problema, expresándolo en la siguiente pregunta de investigación: ¿Cuáles son los principales constructos y variables en el ambiente virtual de aprendizaje, desde la perspectiva de la técnica de modelos de ecuaciones estructurales con la fin de localizar su impacto en la producción científica?

De acuerdo a lo anterior, el planteamiento del problema se justifica primeramente por identificar las teorías relacionadas con los modelos de ecuaciones estructurales. Respecto a los beneficios de su consecución, se encuentran los de informar sobre un grupo de constructos que intervienen en la enseñanza aprendizaje, para conocer la causalidad de sus relaciones. La ventaja será la de poder generar una reflexión, al responder a las necesidades de un modelo virtual. Los beneficiados, serán principalmente los alumnos y docentes, en consideración a que pueden identificar la aplicación causal de los constructos en modelos de educación a distancia. El objetivo que guio el trabajo, fue el de identificar las principales publicaciones, así como los constructos en ellas incluidos para explicar el vínculo entre lo virtual, el aprendizaje y su parte estructural.

Como se indicó, el interés se centra en explicar por qué ocurre la asociación y en qué condiciones se manifiesta. Por otra parte, Bunge (2000) señala que toda explicación racional de un hecho, es una secuencia hipotético-deductiva la cual contiene una información y una generalización. En este sentido, la delimitación o alcance del problema, tiene como referente a las teorías investigadas. Es decir, una directriz gestada por el propio problema, con relación al objeto de estudio (Schmelkes, 2007). Por lo anterior, se propuso la siguiente hipótesis: existen constructos que fundamentan el ambiente virtual de aprendizaje, desde la perspectiva del procesamiento de información y de la técnica del modelo de ecuaciones estructurales, de tal forma que existen evidencias de publicaciones científicas que reportan su frecuente aplicación.

Constructos y variables del ambiente virtual de aprendizaje, desde la perspectiva del modelo de ecuaciones estructurales. Alfredo Ángel Ramírez-Carbajal.

Página 2 de 25 
Por lo que respecta a la revisión de la literatura, se presentan los resultados del análisis de la producción científica de dos temáticas: artículos relacionados con el entorno virtual de aprendizaje y artículos asociados con el modelo de ecuaciones estructurales de los últimos años. Para tener una primera aproximación al estado de la investigación, se ingresó a bases de datos para cuantificar la cantidad de registros correspondiente al periodo 2000-2015, utilizando en tal propósito un grupo de palabras clave relacionadas con el objeto de estudio. Durante el desarrollo de la actividad se logró identificar el volumen de artículos y documentos en cada una de las citadas temáticas. Las bases de datos consultadas fueron las siguientes: EBSCO, Web of Science, Proquest, ScienceDirect, Redalyc, Springerlink y por ultimo Taylor y Francis.

Los datos resultantes de la exploración (véase tabla 1) con base a las palabras clave, tomadas como criterio exhaustivo de selección, se presentan en las siguientes respectivas columnas: $1=$-learning, $2=$ Structural equation model, $3=$ Educación a distancia, $4=$ Modelo de ecuaciones estructurales, $5=$ Entorno virtual aprendizaje y $6=$ Ambiente virtual de aprendizaje. En todos los casos la búsqueda fue con las mismas palabras clave.

\begin{tabular}{|lrrrrrr|}
\hline \multicolumn{1}{|c}{ Nombre } & $\mathbf{1}$ & $\mathbf{2}$ & $\mathbf{3}$ & $\mathbf{4}$ & $\mathbf{5}$ & $\mathbf{6}$ \\
\hline EBSCO & 17841 & 5996 & 1545 & 381 & 37 & 25 \\
\hline Web of Science & 3291 & 2753 & 65 & 8 & 1 & 11 \\
\hline Proquest & 18462 & 420134 & 1811 & 630 & 1034 & 1213 \\
\hline ScienceDirect & 943937 & 466007 & 2418 & 426 & 306 & 162 \\
\hline Springerlink & 22286 & 310068 & 1712 & 17 & 16 & 18 \\
\hline Taylor \& Francis & 319947 & 108461 & 566 & 94 & 50 & 14 \\
\hline Redalyc & 2599 & 228739 & 381291 & 236162 & 114667 & 181714 \\
\hline
\end{tabular}

Tabla 1. Bases de datos.

Fuente: elaboración propia, con base en los datos obtenidos (corresponden a la consulta realizada el 30 de junio de 2015) en los sitios Web de las respectivas bases de datos.

Como resultado del trabajo de exploración anterior, se observa la producción científica en dos ejes temáticos: ambiente virtual de aprendizaje y modelo de ecuaciones estructurales.

Contextualización del ámbito educativo. En esta sección se describen los principales conceptos, teorías y paradigmas. De acuerdo con Hernández (2004) educar es un fenómeno sociocultural muy complejo y para su completo estudio y análisis, son 
necesarias la participación y la colaboración de múltiples disciplinas, que expliquen sus distintas dimensiones y contextos. En este sentido, las ciencias de la educación comprenden todas las disciplinas interesadas en el estudio científico, de los distintos aspectos de la educación, en sociedades y culturas determinadas. Las ciencias de la educación, según Mialaret en Hernández (2004) pueden clasificarse en tres grupos:

a. Las referidas al estudio de las condiciones generales o locales de la educación.

b. Las concernientes a estudiar las situaciones y hechos educativos.

c. Las dirigidas al estudio de la evolución o de la reflexión sobre la educación o al desarrollo.

Es difícil establecerle límites exclusivos de acuerdo con la clasificación y por supuesto en ocasiones son rebasados estos.

Por otra parte Salinas Ibáñez citado por Martínez y Prendes (2007) conceptualiza a la educación a distancia, como aquellas situaciones de aprendizaje en donde normalmente el maestro y el alumno no se encuentran físicamente presentes allí donde la educación tiene lugar y como consecuencia directa de la separación, el aprendizaje a distancia se basa en el uso de diferentes medios técnicos o físicos como elementos portadores de la información y la comunicación, convirtiéndose en la principal característica de los sistemas de educación a distancia o también llamado virtual.

Cabe destacar la existencia de distintos enfoques o corrientes de pensamiento, los cuales pueden ayudar a abordar el tema de educación a distancia. Keegan (1986) en Martínez y Prendes (2007) mencionan la teoría de la autonomía y la independencia o la teoría de la industrialización de la enseñanza (centrada en las funciones de la institución como productora de materiales de aprendizaje), teoría de la interacción y la comunicación (centradas en la función de las instituciones) para posibilitar a los estudiantes experiencias de aprendizaje con base a la comunicación (cuando los materiales ya han sido creados y distribuidos). En este mismo sentido, la teoría del procesamiento de la información, considera que la actividad principal para alcanzar la toma de consciencia es la auto-monitorización, donde el sujeto realiza ajustes de sus acciones para alcanzar las metas que se proponen (Panadero y Alonso-Tapia, 2014).

Por otra parte, los paradigmas en educación han existido desde los albores de la Psicología de la Educación y con un claro interés por la problemática educativa. De manera puntual se relaciona a los siguientes: El paradigma conductista, reconociéndose el trabajo de Skinner y sus seguidores. El paradigma de orientación cognitiva, destacándose los trabajos de Ausbel, Bruner y Wittrock. El paradigma humanista, tiene entre sus representantes a Maslow y Rogers. El Paradigma Psicogenético Piagetano, propuesto por Piaget y sus seguidores. El paradigma Sociocultural, impulsado por Cole, Wertsch y Rogoff (Hernández, 2004). Estos paradigmas se han aplicado al contexto educativo y con ello, se ha dado un impulso decisivo al desarrollo de la psicología de la educación, pues ha posibilitado el surgimiento de aportaciones teóricas e instrumentos 
metodológicos y tecnológicos; beneficiando de esto en forma particular: los materiales didácticos. De acuerdo a las teorías enunciadas anteriormente, sus fortalezas y críticas en ellas han existido (véase tabla 2) desde hace años, por lo que en forma breve se presenta una relación de algunas de ellas.

\begin{tabular}{|c|c|c|}
\hline Teoría & Fortalezas & Criticas \\
\hline Operante & Retraso de la gratificación & Origen del auto-refuerzo \\
\hline Fenomenológica & Papel de la auto-identidades & $\begin{array}{l}\text { Definición, evaluación } \\
\text { validación de las auto } \\
\text { identidades }\end{array}$ \\
\hline $\begin{array}{l}\text { Procesamiento de } \\
\text { la información }\end{array}$ & $\begin{array}{l}\text { Memorización a través de ciclos } \\
\text { de retroalimentación }\end{array}$ & $\begin{array}{l}\text { Ciclos de retroalimentación } \\
\text { negativos vs. Positivos }\end{array}$ \\
\hline Socio cognitiva & $\begin{array}{l}\text { Metas cognitivas y expectativas. } \\
\text { Modelado social }\end{array}$ & $\begin{array}{l}\text { Auto-eficacia: redundante o de } \\
\text { alcance limitado }\end{array}$ \\
\hline Volicional & Persistencia y atención & $\begin{array}{l}\text { Delimitación de la volición y la } \\
\text { motivación }\end{array}$ \\
\hline Vygotskiana & $\begin{array}{lcc}\text { Auto-verbalizaciones } & \text { y } & \text { el } \\
\text { desarrollo del dialogo social } & \end{array}$ & $\begin{array}{l}\text { Auto verbalizaciones vs. Co- } \\
\text { constructivismos social como } \\
\text { métodos de enseñanza de la } \\
\text { auto regulación }\end{array}$ \\
\hline Constructivista & Teorías personales y estrategias & $\begin{array}{l}\text { Papel de la disonancia } \\
\text { cognitiva vs. Contexto situado }\end{array}$ \\
\hline
\end{tabular}

Tabla 2. Fortalezas y críticas de las teorías del aprendizaje.

Fuente: Panadero y Alonso-Tapia (2014).

De acuerdo con Garrison y Shale en Basabe (2007) la educación a distancia es la comunicación educativa entre maestro y los estudiantes, ocurriendo esta de manera síncrona o asíncrona. Para lo cual, es necesario su implementación por medio de un determinado modelo.

Además, en la presente década se considera a la educación a distancia, como el resultado de factores determinantes, entre los cuales se encuentran: la renovación permanente de los conocimientos y técnicas, el impacto político-social de la extensión educativa, el fenómeno pedagógico del perfeccionamiento de la enseñanza, los avances de las tecnologías de la información y las comunicaciones y la atención a la demanda de población trabajadora. Por consiguiente, los mundos virtuales, las realidades artificiales 
y los ciberespacios suponen una revolución en la educación.En esta sección se describieron elementos teóricos - conceptuales afín de contextualizar la investigación. En la siguiente, se presenta la primera de cuatro teorías que fundamentan el presente documento.

Teoría de la acción razonada. La teoría de la acción razonada (TRA, Theory of Reasoned Action), fue propuesta por Fishbein y Ajzen (1975) y es publicada como resultado de su trabajo en el área de la psicología (véase figura 1). Es el antecedente de otra teoría, denominada modelo de aceptación de la tecnología y dada a conocer en 1985. Según sus autores, la TRA postula las relaciones entre actitudes, convicciones, presión social, intenciones y conducta. Es decir, la conducta del sujeto está condicionada por su intención.

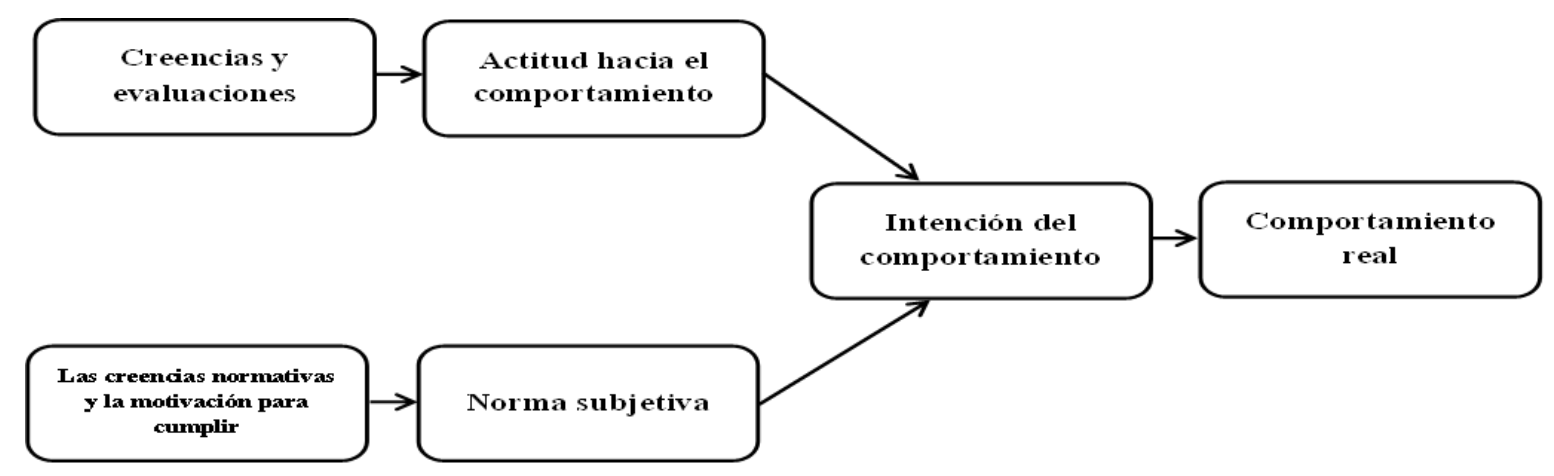

Figura 1. Teoría de la acción razonada. (Fishbein y Ajzen, 1975).

Esta teoría se sustenta en la comprensión de la conducta del sujeto, por consiguiente se requiere conocer cuál es su intención (respecto a si desea o no hacer alguna acción en particular) entendiéndose a las intenciones como un balance entre dos circunstancias: la actitud (relacionada con lo que se cree se debe hacer) y la presión del grupo (es la percepción del sujeto respecto a cómo los otros piensan se debe hacer).

En otras palabras, la teoría de la acción razonada establece la intención de realizar o no realizar una acción y se manifiesta con una conducta. De acuerdo con lo anterior, la intención de la conducta de una persona está determinada por alguno o algunos de los siguientes aspectos:

a. Percepción de autoeficacia para la implementación de dicha conducta.

b. Los efectos derivados de su conducta, así como la posibilidad de las mismas.

c. La relevancia de esos efectos en la persona.

d. El comportamiento esperado del sujeto —según su percepción- las personas significativas de su entorno.

e. La importancia que el sujeto da a la opinión de otras personas.

f. Valoración de las oportunidades y facilidades externas para la manifestación de la conducta.

Teoría del modelo de aceptación de la tecnología. La teoría del modelo de aceptación de tecnología fue propuesta por Fred Davis, también conocida como TAM (Technology Acceptance Model), ha captado la atención en la comunidad de 
Sistemas de Información (SI) y se desarrolló específicamente para explicar la aceptación del usuario, respecto de la tecnología informática (véase figura 2) siendo esencial su comprensión para estudios relacionados con la aceptación de la tecnología. La argumentación esencial propuesta por Davis (1985) está referida al Uso del sistema como una respuesta para ser explicada o predicha por la motivación del usuario, a su vez, está directamente influenciado por un estímulo externo, el cual se encuentra vinculado a las características del sistema y sus propias capacidades. Al momento de darse a conocer, fue definida como el estado psicológico de un individuo con respecto a la utilización voluntaria o involuntaria de una tecnología en particular.

TAM propone que los elementos: utilidad percibida, facilidad de uso y percepción de la tecnología sean variables de predicción de la actitud del usuario hacia el uso de la tecnología, así como de la posterior intención de comportamiento. Esto daría como resultado un determinado uso real. Tiene por finalidad explicar las causas de aceptación de la tecnología por los usuarios y plantea que las percepciones de un individuo; respecto de la utilidad y la facilidad de uso de un sistema de información, son concluyentes para valorar su intención al usar un sistema. Por consecuencia, su propósito primario es el de indagar el impacto de las variables externas en dos aspectos: la utilidad percibida y la facilidad de uso percibida, para predecir el uso de las Tecnologías de la Información y la Comunicación.

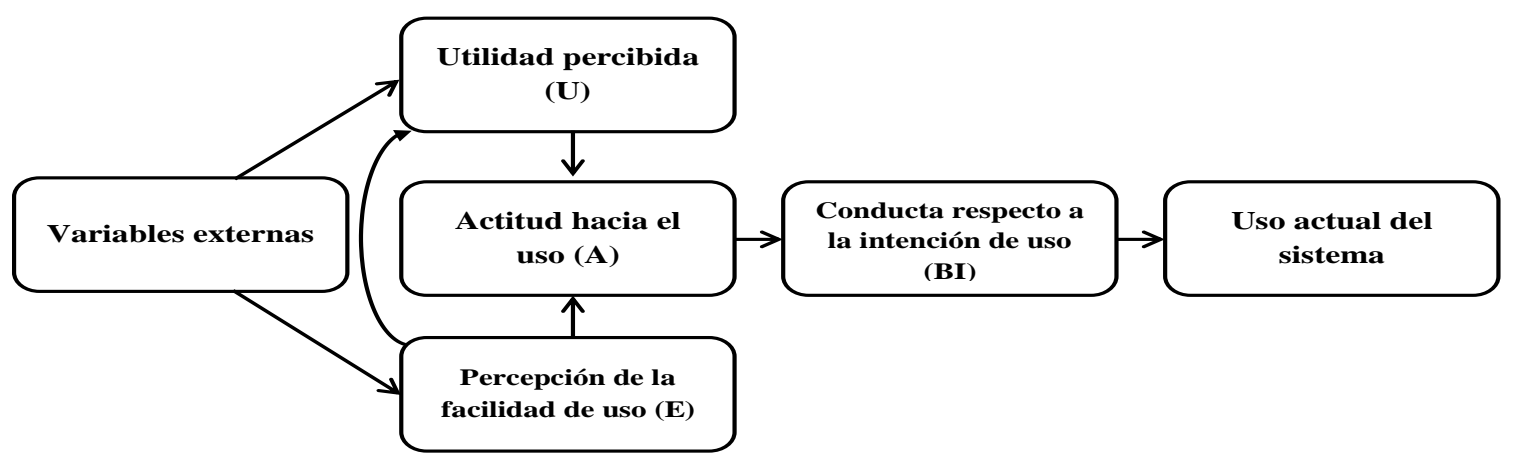

Figura 2. Modelo de aceptación de la tecnología (Davis, 1985: 24).

La TAM se ha aplicado en numerosos estudios de la tecnología de la información, como procesadores de texto, aplicaciones de hoja de cálculo, correo electrónico, navegadores web, sitios web, etcétera. Por último, se ha demostrado que es un modelo de investigación apropiado para analizar el uso de las tecnologías, ya sea como parte principal en la educación a distancia o como complemento al proceso tradicional de aprendizaje.

Teoría del modelo de éxito de los sistemas de información. La teoría del modelo de éxito de los sistemas de información (ISSM, por sus siglas en el idioma ingles), fue propuesto inicialmente por DeLone y McLean en el año de 1992, en su artículo publicado Information system Success: the quest for the dependent variable. DeLone y McLean (1992) señalan la importancia de realizar la medición del éxito en los SI o su eficacia para comprender y valorar su calidad, respecto a las acciones relacionadas con su administración e inversiones en los SI (véase figura 3). Como parte del trabajo 
exploratorio, se logró identificar que desde la publicación por primera vez en su artículo, cerca de trescientos artículos en revistas especializadas se han referido al uso del modelo de éxito. La gran popularidad de este modelo es una fuerte evidencia para continuar su aplicación. Diez años más tarde (DeLone y McLean, 2003) revisan por primera vez su modelo y publican un artículo en donde, partiendo de los elementos estrechamente vinculados a la evaluación del éxito de un sistema de información y para atender las opiniones y críticas de académicos y profesionales. El objetivo principal de este modelo es identificar los elementos que contribuyen al éxito de los sistemas de información.

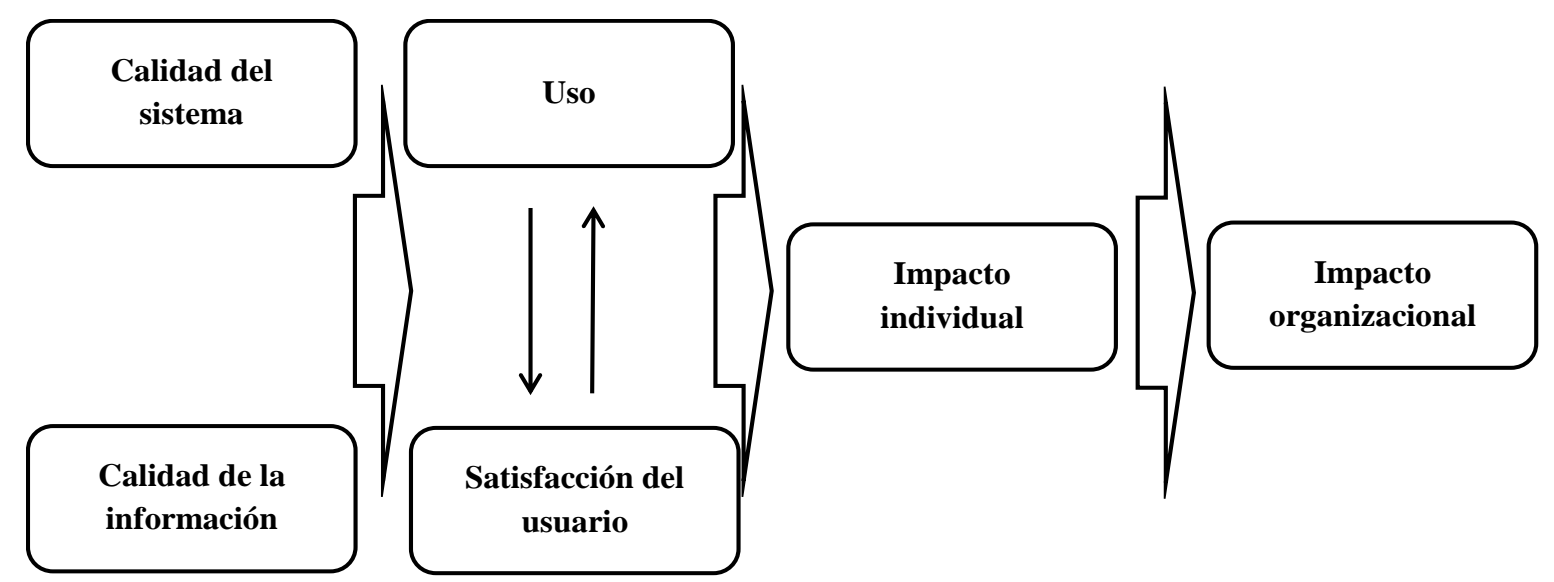

Figura 3. Modelo de éxito de los sistemas de información (DeLone y McLean, 2003:

87.).

Los autores, proponen el tipo de influencia de la calidad de la información y la calidad del sistema, en el uso y en la satisfacción del usuario. El uso y la satisfacción del usuario a su vez afectan al impacto individual, y éste influye en el impacto organizacional. El modelo se construyó sobre la base de la naturaleza lineal del proceso y los autores especifican, que cuando se utilice el modelo, el objetivo debería ser el análisis de las seis dimensiones en forma interrelacionada, donde se observa la dependencia de unas, respecto de otras, en lugar de preocuparse por las relaciones causales entre ellas. Conforme pasa el tiempo, estos dos autores realizan adecuaciones y aproximadamente una década después dan a conocer la segunda revisión (véase figura 4), del modelo de éxito de los sistemas de información. 


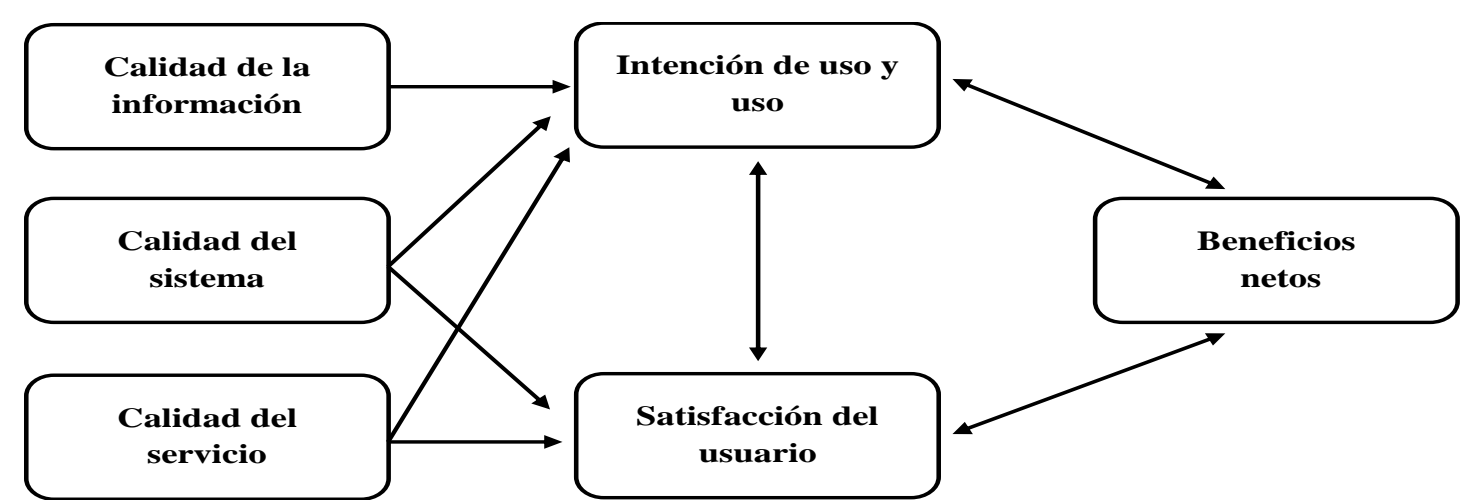

Figura 4. Modelo de éxito de los sistemas de información. Segunda revisión (DeLone y McLean, 2003: 9).

En el modelo renovado no se consideran separadamente los impactos individual y organizacional, y otros nuevos tipos de impacto (Seddon, 1997) donde el uso de un sistema de información es referida a una forma de comportamiento, más que una medida de éxito, y propone sustituir en el modelo la variable uso, por la de utilidad percibida y se le considera como una medida general de la percepción de los beneficios netos del uso de un sistema de información. Respecto al impacto en el grupo de trabajo o el impacto en el consumidor, se utiliza el término beneficios netos para representar a todas las medidas de resultados posibles, con objeto de simplificar el modelo.

Además, se admite en el modelo original a la variable Uso, sin tener en cuenta la complejidad real, ni las dificultades en la interpretación (obligatorio o voluntario, informado o mal informado, eficaz o ineficaz).

Aunque en la segunda versión del modelo del éxito de los sistemas de información continúan relacionando sus componentes en un sentido lineal, se incluyen expresamente relaciones de retroalimentación sin especificar el signo de las relaciones. Continuando con Seddon, P. (1997) la causalidad se expresa en la relación, como la que existe entre el uso y la satisfacción del usuario. El término uso, debe preceder a la satisfacción del usuario en un sentido de proceso lineal, pero una experiencia positiva con el mismo, dará lugar a una mayor satisfacción de quien lo usa en un sentido causal.

Esto lo podemos ver cuando los autores, refiriéndose a su segunda versión, comentan respecto a la "inclusión de flechas" para mostrar las relaciones propuestas entre las dimensiones del éxito en un sentido lineal de un proceso, pero no se muestra el signo positivo o negativo de las relaciones en un sentido causal. La propiedad de la relación de causalidad debe ser supuesta en el contexto de cada caso particular (Hoyle, 2012). Una disminución de la calidad se traduce en una disminución del Uso y de la Satisfacción del usuario, lo que implica una disminución de los Beneficios netos (véase figura 5). Es decir, una disminución de la calidad ocasiona, una disminución del beneficio. 


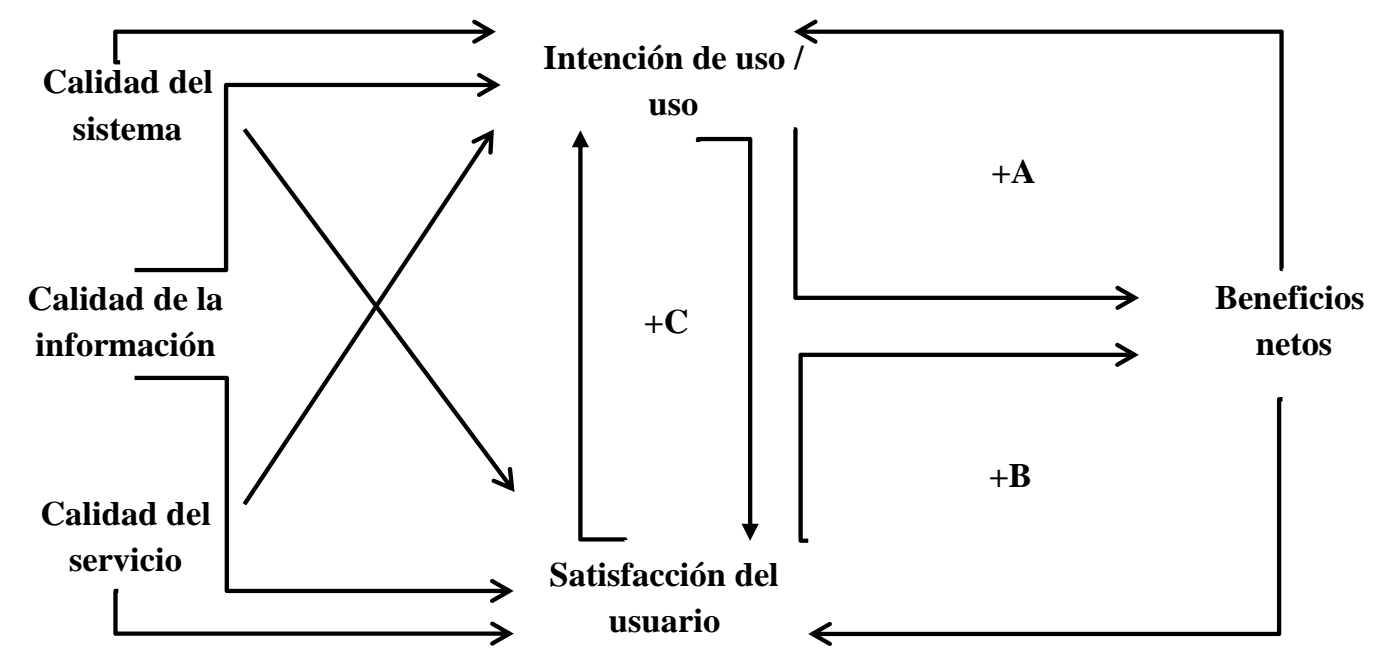

Figura 5. Diagrama causal. (Morlán, 2010: 141).

Los flujos se muestran en primer lugar en el lado superior derecho: entre los Beneficios netos y la Intención de uso / Uso (representado como +A). En segundo lugar: entre Beneficios netos y la Satisfacción del usuario, (representado como +B). Por último, entre la Intención de uso / Uso con el de la Satisfacción del usuario (representado como $+\mathrm{C})$. Los flujos circulares de $+\mathrm{A}$ y $+\mathrm{B}$ muestran como una vez que el usuario experimenta los beneficios netos del sistema, estará frecuentemente más dispuesto a utilizar el sistema, y también se mostrará más satisfecho con el uso del sistema.

El flujo circular de $+\mathrm{C}$, puede parecer reiterativo, especialmente con la relación positiva entre el Uso y la Satisfacción del usuario. La relación ya existía en el modelo original y la explicación se encuentra en la variable experiencia. El usuario cuando adquiere experiencia por la utilización del sistema, simultáneamente amplía las posibilidades de uso del mismo. A su vez incrementa la Satisfacción del usuario. Por último, en el siguiente apartado se describe (por su enfoque estadístico), la teoría de los modelos de ecuaciones estructurales.

Teoría del modelo de ecuaciones estructurales. Aquí, se describe las características elementales de la teoría de modelo de ecuaciones estructurales (SEM, Structural Equation Model) la cual se puede utilizar en cálculos con más de dos variables (Coenders, Batista y Saris, 2005).

De acuerdo con Kaplan (2009) sus aspectos técnicos comienzan — sin conocerse con este nombre al inicio - a ser planteados a partir de 1918. En 1934, Wright, publica el trabajo modelo de trayectoria (path analysis) sobre las relaciones de tamaño en mediciones óseas. En dicho trabajo, Wright señalaba "éste no era un método para descubrir causas, sino más bien era un método aplicado a modelos causales ya formulados con base a un conocimiento y consideración teórica". La técnica permitía descomponer la varianza y covarianza de las variables involucradas, en función de los parámetros de un sistema de ecuaciones simultáneas y tenía como objetivo analizar el efecto directo e indirecto entre estas variables.

Constructos y variables del ambiente virtual de aprendizaje, desde la perspectiva del modelo de ecuaciones estructurales. Alfredo Ángel Ramírez-Carbajal. 
Es decir, se trabaja a partir de constructos (Brown, 20006) para más adelante evaluar la calidad de dicha medición por medio de la correlación (Hoyle, 2012). La teoría tiene fundamentos estadísticos. Con frecuencia se recurre a un modelo, para describir la relación entre una variable dependiente $(\mathrm{Y})$ y una variable independiente $(\mathrm{X})$ que se estima pudiera tener efectos en Y.

Obsérvese como entre las variables independientes puede existir la influencia recíproca entre ellas mismas (véase figura 6), haciéndose más complejo el modelo y por consecuencia se necesitaría más de una ecuación para describir toda la estructura. De acuerdo a lo anterior y con los datos, se estiman los parámetros del modelo, se verifica su validez y precisión para que, en suma se rechace o no se rechace la relación propuesta.

De esta forma, uno de los aspectos fundamentales a investigar en estos trabajos, son las posibles asociaciones entre variables observables (directamente detectadas o medibles) o entre variables observables y variables latentes (un conjunto de variables relacionadas lógicamente por una teoría) con el fin de identificar relaciones de causalidad (Batista y Coenders, 2000; Hoyle, 2012). Por consecuencia, esto conduce a una tarea cuidadosa en el sentido de establecer con claridad la relación entre las distintas variables del modelo.

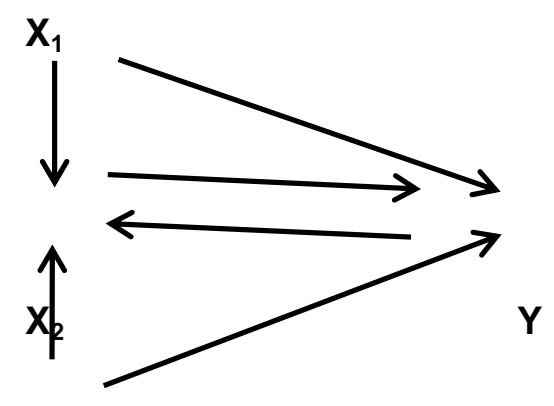

Figura 6. Diagrama básico (Silva y Schiattino, 2008: 106).

En el ámbito estadístico, la causalidad puede plantear relaciones entre variables de interés y dotarlas de direcciones lógicas, para evaluar la fuerza de tales asociaciones. Esto es conocido como análisis de trayectoria o por sus siglas en inglés como path analysis (Batista y Coenders, 2000).

Por su parte, Silva y Schiattino (2008) señalan que combinando algunas técnicas estadísticas, tales como: los sistemas de ecuaciones lineales, el análisis de trayectorias o análisis factorial exploratorio y confirmatorio, es posible describir gráfica y analíticamente las relaciones existentes entre las variables observables y las no observables (tomando en cuenta la dirección de cada una de tales relaciones). A partir de una determinada muestra, se pueden estimar tales relaciones y juzgar su importancia, simplificando paulatinamente los diagramas iniciales hasta obtener un modelo apegado a la realidad. Para tal fin, existe en la actualidad software como LISREL, AMOS o PLS los cuales se usan en apoyo del trabajo estadístico.

Constructos y variables del ambiente virtual de aprendizaje, desde la perspectiva del modelo de ecuaciones estructurales. Alfredo Ángel Ramírez-Carbajal.

Página 11 de 25 
Por su parte, Kaplan (2009) define a este tipo de modelado, como una metodología que busca representar hipótesis sobre datos estadísticos, derivados de mediciones empíricas en términos de un menor número de parámetros estructurales, definidos por un modelo subyacente de constructos.

De acuerdo con Ruiz, Pardo y San Martin (2010) los modelos estructurales son una familia de modelos estadísticos multivariantes y sus fundamentos teóricos estiman el efecto y las relaciones entre un grupo de variables.

Para tal fin, en el desarrollo de estos modelos, se incorporan variables latentes (es el conjunto de variables observables) y variables observables (también conocidas como medidas), cuyos datos - mediciones - provienen directamente del objeto observado.

Como se observó en las secciones precedentes, se hace patente el desarrollo de la fundamentación teórica y de su impacto en los ámbitos de la Educación, Medicina, Sociología, Psicología y otros. De acuerdo al trabajo exploratorio inicial y a la revisión de la literatura se logró identificar las siguientes aplicaciones: en la educación se realizan estudios sobre motivación, educación a distancia o desempeño académico del estudiante, por mencionar algunos y su uso se encuentra documentado en una gran cantidad de artículos registrados en revistas científicas.

\section{Métodos y materiales}

En este apartado se describe el procedimiento de la investigación y el cual consta de tres etapas. En la primera se aplica una serie de criterios (más adelante descritos) para obtener la población y posteriormente derivar en una muestra de artículos. En la segunda, se identifican los constructos de esta. En la tercera, se obtiene un grupo de variables. El método fue dirigido hacia la investigación documental.

Con base a la fundamentación teórico-conceptual descrita con anterioridad, se realizaron actividades para aproximarse a la frontera del conocimiento.

A partir de un conjunto de aproximadamente 1175 artículos relacionados con el objeto de estudio, se logró reducir la selección previa por medio de los criterios de: fecha de publicación, temática, instrumento, ambiente virtual de aprendizaje, tipo de modelo y técnica estadística.

Con estas acciones se conforma la muestra de 421 artículos publicados en revistas arbitradas e indexadas, con la finalidad de identificar las investigaciones publicadas en revistas que cumplieran con dichos criterios.

De acuerdo a la anterior descripción de la secuencia de etapas, se obtuvieron con todas ellas la muestra representativa. En el siguiente apartado, se presentan los resultados.

\section{Resultados}

En este apartado se explican los resultados. En primer lugar se presenta la correspondencia entre la fundamentación teórica y un grupo de artículos (véase tabla 3)

Constructos y variables del ambiente virtual de aprendizaje, desde la perspectiva del modelo de ecuaciones estructurales. Alfredo Ángel Ramírez-Carbajal.

Página 12 de 25 
los cuales fueron seleccionados de la muestra con determinados criterios.

Para tal efecto, realiza una exhaustiva selección de trabajos de investigación para identificar las relaciones entre los autores y las teorías citadas, así como del tipo de recolección de datos, técnica de análisis y tipo de sistema en sus investigaciones. En la siguiente tabla, se identifican las columnas de la siguiente forma:

1 = teoría ISSM, 2 = teoría TAM y 3 = otra teoría, $4=$ software usado en la investigación (Amos / Lisrel / PLS), 5 = tipo de recolección de datos (encuesta / en línea / telefónica / entrevista / con aplicación / prototipo), 6 = técnica de análisis (análisis de regresión múltiple) y $7=$ otra técnica (ANOVA, análisis factorial, experimento fuera de línea, y experimento Online) véase tabla 3.

\begin{tabular}{|c|c|c|c|c|c|c|c|}
\hline Autores & $\mathbf{1}$ & 2 & 3 & 4 & 5 & 6 & Tipo de sistema. \\
\hline $\begin{array}{l}\text { Shu-Sheng, L. et al. } \\
(2003)\end{array}$ & $\bullet$ & $\bullet$ & & & - & • & De calidad \\
\hline $\begin{array}{l}\text { Chao-Min, Ch. et al. } \\
\text { (2005) }\end{array}$ & & & $\bullet$ & $\bullet$ & • & & $\begin{array}{l}\text { La percepción de facilidad de uso y la } \\
\text { calidad percibida }\end{array}$ \\
\hline $\begin{array}{l}\text { Shu-Sheng, L. et al. } \\
(2006)\end{array}$ & $\bullet$ & $\bullet$ & & & $\bullet$ & • & De calidad \\
\hline Roca, J. et al. (2006) & $\bullet$ & $\bullet$ & $\bullet$ & $\bullet$ & $\cdot$ & & De calidad y calidad de la información \\
\hline Ya-Ching, L. (2006) & & $\bullet$ & & & • & - & $\begin{array}{l}\text { Percepción de la calidad del contenido y } \\
\text { atributos del curso }\end{array}$ \\
\hline $\begin{array}{l}\text { Holsapple, C. y A. } \\
\text { Lee-Post (2006) }\end{array}$ & $\bullet$ & & & & • & & $\begin{array}{l}\text { Porcentaje de distribución. Sistema: de } \\
\text { calidad y calidad de la información }\end{array}$ \\
\hline Hsiu-Fen, L. (2007) & $\bullet$ & & & & $\bullet$ & & De calidad y calidad de la información \\
\hline $\begin{array}{l}\text { Yeung, P. y E. } \\
\text { Jordan, (2007) }\end{array}$ & $\bullet$ & $\bullet$ & $\bullet$ & - & $\bullet$ & & De calidad y calidad de la información \\
\hline Poelmans, S. (2008) & $\bullet$ & $\bullet$ & & $\bullet$ & $\bullet$ & & De calidad y calidad de la información \\
\hline $\begin{array}{l}\text { Su-Chao, Ch. y T. } \\
\text { Feng-Cheng (2008) }\end{array}$ & & $\bullet$ & $\bullet$ & $\bullet$ & - & & Percepción de la calidad del sistema \\
\hline $\begin{array}{l}\text { Mueller, D. y V. } \\
\text { Zimmermann }\end{array}$ & $\bullet$ & $\bullet$ & & & & & $\begin{array}{l}\text { Conceptual. Sistema: de calidad y calidad } \\
\text { de la información }\end{array}$ \\
\hline
\end{tabular}

Constructos y variables del ambiente virtual de aprendizaje, desde la perspectiva del modelo de ecuaciones estructurales. Alfredo Ángel Ramírez-Carbajal. 


\begin{tabular}{|c|c|c|c|c|c|c|c|c|}
\hline Autores & $\mathbf{1}$ & 2 & 3 & 4 & 5 & 6 & 7 & Tipo de sistema. \\
\hline$(2009)$ & & & & & & & & \\
\hline $\begin{array}{l}\text { Yi-Shun, W. et al. } \\
\text { (2007) }\end{array}$ & $\bullet$ & $\bullet$ & & $\bullet$ & $\bullet$ & & & De calidad y calidad de la información \\
\hline $\begin{array}{l}\text { Tobing, V. et al. } \\
\text { (2008) }\end{array}$ & & $\bullet$ & & & & $\bullet$ & & Adaptabilidad del sistema \\
\hline Arbaugh, J. (2000) & & $\cdot$ & & & $\bullet$ & & & $\begin{array}{l}\text { Offline. Análisis de regresión. Percepción } \\
\text { de la iteración del curso, flexibilidad } \\
\text { percibida (tiempo, localización, métodos). }\end{array}$ \\
\hline $\begin{array}{l}\text { Hong, W. et al. } \\
\text { (2002) }\end{array}$ & & • & & • & • & & & $\begin{array}{l}\text { Diseño de pantalla, relevancia, } \\
\text { terminología }\end{array}$ \\
\hline Yi-Shun, W. (2003) & & & - & & • & & - & $\begin{array}{l}\text { Interface de aprendizaje, comunidad de } \\
\text { aprendizaje, contenido personalizado. }\end{array}$ \\
\hline $\begin{array}{l}\text { Su-Houn, L. et al. } \\
(2005)\end{array}$ & & $\cdot$ & • & & • & & - & $\begin{array}{l}\text { Material de educación a distancia, tipos de } \\
\text { presentación, (texto- audio, Audio-video, } \\
\text { texto-audio-video). }\end{array}$ \\
\hline $\begin{array}{l}\text { Goon, L. et al. } \\
\text { (2005) }\end{array}$ & & • & & & - & $\bullet$ & & $\begin{array}{l}\text { Diseño de video, navegación, } \\
\text { terminología }\end{array}$ \\
\hline $\begin{array}{l}\text { Pituch, K. y L. Yao- } \\
\text { Kuei (2006) }\end{array}$ & & $\bullet$ & & • & & & • & $\begin{array}{l}\text { Funcionalidad del sistema, interactividad } \\
\text { del sistema, respuesta del sistema }\end{array}$ \\
\hline $\begin{array}{l}\text { Fong-Ling, F. et al. } \\
(2007)\end{array}$ & & • & & • & $\cdot$ & & - & $\begin{array}{l}\text { Funcionalidad del sistema, interface de } \\
\text { diseño }\end{array}$ \\
\hline $\begin{array}{l}\text { Pei-Chen, S. et al. } \\
\text { (2008) }\end{array}$ & & & • & & $\cdot$ & - & & $\begin{array}{l}\text { Flexibilidad del curso (tiempo, } \\
\text { localización, método) calidad de la } \\
\text { tecnología, calidad del curso }\end{array}$ \\
\hline $\begin{array}{l}\text { Martínez-Torres, } \\
\text { M. et al. (2008) }\end{array}$ & & $e^{\circ}$ & & $\bullet$ & $\cdot$ & & & $\begin{array}{l}\text { Accesibilidad, comunicación, } \\
\text { retroalimentación, interactividad y } \\
\text { control, desempeño, adaptación del } \\
\text { usuario, usuario, herramientas, formato }\end{array}$ \\
\hline $\begin{array}{l}\text { Sahin, I. y M. } \\
\text { Shelley (2008) }\end{array}$ & & $\bullet$ & & $\bullet$ & $\bullet$ & & & Flexibilidad \\
\hline
\end{tabular}




\begin{tabular}{|c|c|c|c|c|c|c|c|}
\hline Autores & 1 & 2 & 3 & 4 & 5 & $6 \mathbf{7}$ & Tipo de sistema. \\
\hline $\begin{array}{l}\text { Nov, O. y C. Ye } \\
(2008)\end{array}$ & & • & & & $\bullet$ & $\bullet$ & Diseño de pantalla, relevancia \\
\hline Arbaugh, J. (2002) & & & $\bullet$ & & $\cdot$ & $\bullet$ & $\begin{array}{l}\text { Flexibilidad del curso (tiempo, } \\
\text { localización, método). Flexibilidad del } \\
\text { programa (tiempo, localización, método) }\end{array}$ \\
\hline Cho, V. et al. (2009) & & • & $\bullet$ & $\bullet$ & $\bullet$ & & $\begin{array}{l}\text { Percepción de intención de uso, } \\
\text { percepción del diseño }\end{array}$ \\
\hline $\begin{array}{l}\text { Johnson, R. et al. } \\
\text { (2009) }\end{array}$ & & & $\bullet$ & & $\bullet$ & $\bullet$ & Media síncrono \\
\hline $\begin{array}{l}\text { Thong, J. et al. } \\
\text { (2002) }\end{array}$ & & • & & & $\bullet$ & $\bullet$ & $\begin{array}{l}\text { Diseño de la navegación en pantalla, } \\
\text { relevancia terminología }\end{array}$ \\
\hline $\begin{array}{l}\text { Jen-Her, W. et al. } \\
\text { (2008) }\end{array}$ & & $\bullet$ & & $\bullet$ & $\bullet$ & & $\begin{array}{l}\text { Funcionalidad del sistema y } \\
\text { características de contenido }\end{array}$ \\
\hline
\end{tabular}

Tabla 3. Aplicaciones de las teorías ISSM y TAM.

Fuente: Tabla adaptada del artículo Design characteristics of virtual learning environments: an expert study (Mueller y Strohmeier, 2011: 2509).

En seguida se presentan los constructos empleados en los citados trabajos. Consecuentemente se seleccionan y ordenan. Mismos que son representados en las columnas: 1 = Percepción, 2 = Autoeficacia, $3=$ Instructor, $4=$ Tecnología, $5=$ Actitud, 6 = Calidad, 7 = Curso y $8=$ SEM. Este conjunto de acciones tiene la finalidad de identificar los constructos contenidos en ellos (véase tabla 4).

La rigurosa selección, reporta únicamente documentos que contienen el mayor número de constructos y que corresponden a investigaciones relacionadas con el ambiente virtual de aprendizaje.

Se hace notar, que sus autores se ocuparon en relacionar la mayor cantidad de constructos en sus investigaciones.

\begin{tabular}{|l|c|c|c|c|c|c|c|c|c|}
\hline Autor & 1 & 2 & 3 & 4 & 5 & 6 & 7 & 8 & total \\
\hline Martins L. y W. Kellermanns (2004) & $\bullet$ & $\bullet$ & & $\bullet$ & $\bullet$ & & & $\bullet$ & 5 \\
\hline Su-Chao, Ch. y T. Feng-Cheng (2008) & $\bullet$ & $\bullet$ & & $\bullet$ & $\bullet$ & & & $\bullet$ & 5 \\
\hline
\end{tabular}




\begin{tabular}{|l|c|c|c|c|c|c|c|c|c|}
\hline \multicolumn{1}{|c|}{ Autor } & 1 & 2 & 3 & 4 & 5 & 6 & 7 & 8 & total \\
\hline Picciano, A. (2002) & $\bullet$ & & & & $\bullet$ & & $\bullet$ & & 3 \\
\hline Selim, H. (2007) & & & $\bullet$ & $\bullet$ & $\bullet$ & & & $\bullet$ & 4 \\
\hline Abdalla, I. (2007) & $\bullet$ & & & $\bullet$ & $\bullet$ & & & $\bullet$ & 4 \\
\hline Martínez-Torres, M. et al. (2008) & $\bullet$ & & $\bullet$ & $\bullet$ & $\bullet$ & & $\bullet$ & $\bullet$ & 6 \\
\hline Fernández, R. (2009) & & & & $\bullet$ & & & $\bullet$ & $\bullet$ & 3 \\
\hline Pei-Chen, S. et al. (2008) & $\bullet$ & & $\bullet$ & $\bullet$ & $\bullet$ & $\bullet$ & $\bullet$ & & 6 \\
\hline Wen-Shan, L. y W. Chun-Hsien, (2011) & $\bullet$ & & & $\bullet$ & $\bullet$ & $\bullet$ & & $\bullet$ & 5 \\
\hline Jung-Wan L. (2010) & $\bullet$ & & & & $\bullet$ & $\bullet$ & $\bullet$ & $\bullet$ & 5 \\
\hline Po-An, H. y V. Cho (2011) & $\bullet$ & & & & $\bullet$ & $\bullet$ & & $\bullet$ & 4 \\
\hline Joo, Lim y Kim. (2012) & $\bullet$ & $\bullet$ & $\bullet$ & $\bullet$ & $\bullet$ & & $\bullet$ & $\bullet$ & 7 \\
\hline Sahin, I. y M. Shelley (2008) & & & & $\bullet$ & & & $\bullet$ & $\bullet$ & 3 \\
\hline
\end{tabular}

Tabla 4. Constructos contenidos por artículo

Fuente: elaboración propia, con base a la muestra.

Posteriormente, con la muestra seleccionada y de acuerdo a los constructos, se identificaron las siguientes variables (véase tabla 5), las cuales se describen a continuación:

\begin{tabular}{|lll|}
\hline Nombre & \multicolumn{1}{c|}{ Concepto } & Atributos \\
\hline $\begin{array}{l}\text { Actividad frente } \\
\text { a computadora }\end{array}$ & $\begin{array}{l}\text { Realización de actividades } \\
\text { académicas en la computadora. }\end{array}$ & Trabajo con computadoras \\
& & $\begin{array}{l}\text { Ansiedad frente a la } \\
\text { computadora }\end{array}$ \\
\hline Asesor-tutor & Sujeto que enseña. & Retroalimentación \\
& & Asesor-tutor \\
\hline Curso & Unidad de aprendizaje. & Comunicación \\
& & Sesiones \\
\hline Disposición & $\begin{array}{l}\text { Preparación o modo mostrado ante } \\
\text { el trabajo académico. }\end{array}$ & Motivación \\
& & Intención de utilizar \\
& Cantidad de resultados finales, como & Eficacia \\
& producto del trabajo académico. & Adaptación del usuario \\
& & \\
\hline Efectividad & Actitud & \\
& &
\end{tabular}




\begin{tabular}{|c|c|c|}
\hline Nombre & Concepto & Atributos \\
\hline & & Utilización \\
\hline Ambiente virtual & $\begin{array}{l}\text { Espacio tecnológico donde se realiza } \\
\text { la actividad académica. }\end{array}$ & $\begin{array}{l}\text { Accesibilidad } \\
\text { Interactividad }\end{array}$ \\
\hline Estudio & $\begin{array}{l}\text { Conjunto de actividades académicas } \\
\text { del alumno, para el aprendizaje de la } \\
\text { unidad de estudio. }\end{array}$ & $\begin{array}{l}\text { Hábitos de estudio } \\
\text { Horario de estudio }\end{array}$ \\
\hline $\begin{array}{l}\text { Material } \\
\text { didáctico }\end{array}$ & $\begin{array}{l}\text { Conjunto de documentos en } \\
\text { diferentes formatos y estilos usados } \\
\text { en la unidad de aprendizaje. }\end{array}$ & $\begin{array}{l}\text { Formato y Actividades } \\
\text { Ejemplos } \\
\text { Materiales didácticos }\end{array}$ \\
\hline Percepción & Advertir por medio de los sentidos. & $\begin{array}{l}\text { Uso percibido } \\
\text { Utilidad percibida } \\
\text { Satisfacción percibida }\end{array}$ \\
\hline Sistema & $\begin{array}{l}\text { Elementos interrelacionados de tipo } \\
\text { académico, que tienen como fin la } \\
\text { enseñanza aprendizaje. }\end{array}$ & $\begin{array}{l}\text { Confiabilidad } \\
\text { Calidad } \\
\text { Sistema de aprendizaje }\end{array}$ \\
\hline Tecnología & $\begin{array}{l}\text { Conjunto de recursos de tipo } \\
\text { software y hardware que apoyan la } \\
\text { actividad académica. }\end{array}$ & $\begin{array}{l}\text { Difusión } \\
\text { Tecnología }\end{array}$ \\
\hline
\end{tabular}

Tabla 5. Variables.

Fuente: elaboración propia, con datos de la muestra.

Posteriormente, Se logró identificar las asociaciones entre variables (véase tabla 6) que con mayor frecuencia se emplean en los modelos publicados en los artículos anteriores.

Material didáctico / curso

Hábitos de estudio / curso

Sistema / ambiente virtual 
Actividad frente a la computadora / ambiente virtual.

Curso/instructor-asesor.

Ambiente virtual / instructor-asesor.

Tabla 6. Asociaciones entre constructos

Fuente: elaboración propia, con datos de la muestra.

\section{Discusión de resultados.}

Desde la perspectiva de los modelos de ecuaciones estructurales, estas variables han sido incluidas en distintas investigaciones, como a continuación se explican:

Material didáctico / curso. De acuerdo con Parcerisa (2007) señala que los materiales didácticos son aquellos artefactos que utilizando diferentes formas de representación simbólica u objetos, ayudan a la construcción de conocimientos específicos, dentro de una estrategia de enseñanza más amplia.

Por otra parte Basabe (2007) argumenta que en general, es aceptado que los materiales didácticos cumplan tres funciones básicas: motivadora, portadora de contenidos y estructurante.

Si estos logran aumentar en el alumno el interés por la temática del curso, si presentan la información como una provocación para la reflexión, si es un apoyo para estructurar el contenido y si facilita relacionarlo con sus conocimientos previos, habrán cumplido su cometido dentro del sistema a distancia. Por otra parte, habrán permitido al estudiante construir el conocimiento con un mayor grado de autonomía.

Hábitos de estudio / curso. Con base a esta asociación, Martínez-Otero y Torres (2005) consideran que los elementos que condicionan el rendimiento académico en los distintos niveles de enseñanza son numerosos y constituyen una intrincada red, en la que resulta complejo ponderar la influencia específica de cada uno, dando lugar a que se ha generalizado la idea de que los hábitos de estudio influyen considerablemente en los resultados escolares.

También aclaran que no se deben confundir los hábitos (prácticas constantes de las mismas actividades), con las técnicas (procedimientos o recursos). Unos y otras, empero, coadyuvan en el estudio. Además, revela por una parte, que el hábito de estudiar es necesario, si se quiere progresar en el aprendizaje.

Sistema / ambiente virtual. Estos elementos al interrelacionarse, constituyen el escenario donde se desarrollan condiciones favorables de aprendizaje, considerándose, entre otras: las condiciones necesarias para la implementación del currículo, las relaciones entre instructor-asesor y estudiantes, la organización y disposición temática, las pautas de comportamiento que en ella se desarrollan, el tipo de relaciones que mantienen las personas con los objetos y entre ellas mismas, los roles que se establecen

Constructos y variables del ambiente virtual de aprendizaje, desde la perspectiva del modelo de ecuaciones estructurales. Alfredo Ángel Ramírez-Carbajal.

Página 18 de 25 
y en general las actividades académicas. Estos elementos se relacionan como un entorno sistémico en donde se localizan los materiales didácticos.

Actividad frente a la computadora / ambiente virtual. En este sentido, Stahl, Koschmann, y Suthers (2006) en su obra menciona que el aprendizaje se lleva a cabo en espacios donde haya una alta interacción entre los estudiantes, de tal forma que el aprendizaje, se da a través de la formulación de preguntas, del planteamiento de actividades conjuntas, de enseñar a los demás y de observar como los demás estudiantes aprenden.

Por consiguiente, lograr estimular a los estudiantes para que interactúen de forma efectiva no es algo fácil de lograr, se requiere de una planeación detallada dónde se integre pedagogía y tecnología.

Curso/instructor-asesor. Cabe aclarar que los autores citados en este apartado, les llaman indistintamente mediador, docente, profesor o maestro. En este sentido y al referirse sobre los componentes del sistema de educación a distancia, Basabe (2007) señala que muchos maestros que han desarrollado su actividad docente en la modalidad presencial, consideran que las características de la modalidad a distancia, no debieran ser muy distintas e intentan trasladar sus prácticas y esquemas de una a otra.

En relación con las funciones de los docentes o sus roles en los entornos tecnológicos, García Valcárcel (2003) citado por Basabe (2007) opina que se puede decir que el profesor tiene un papel mediador y facilitador del proceso cognitivo-social. Identifica como funciones del profesor las siguientes: planificación de actividades, selección de contenidos, establecer niveles de aprendizaje, transmitir información, motivar actividades, elaborar contenidos y materiales, orientar, centrarse en el aprendizaje y por último evaluar. De ahí que también se le llame instructor-asesor.

Ambiente virtual / instructor-asesor. En relación a las actividades del tutor, se considera que es un formador y educador que se centra fundamentalmente, en la instrucción de los alumnos y asume funciones de organización de actividades académicas y de motivación, propicia un clima cordial de aprendizaje y es facilitador educativo.

Es decir, favorece las experiencias para el auto-aprendizaje y la construcción del conocimiento. Las actividades se organizan generalmente entre el instructor-asesor y alumno, así como las relaciones intergrupales, preparación y control de la información, gestión del conocimiento y evaluación.

\section{Conclusiones}

En la sección anterior, se discutieron los resultados de la investigación, en la misma se identificaron a los autores, constructos o variables y la aplicación de algunas de las teorías enunciadas previamente. En seguida se presentan las conclusiones.

De acuerdo a la discusión anterior, en la investigación se desarrolló un modelo con base a una serie de factores asociados al ambiente virtual de aprendizaje para analizar las percepciones de los alumnos respecto al aprendizaje en ambientes virtuales. En consideración al uso de los programas de educación a distancia y a través de esta 
investigación, se aportan los resultados de un modelo estructural para que se fortalezca y se contribuya en el uso de esta alternativa educativa.

Los resultados indicaron que los fundamentos se cumplen y a su vez permitieron construir las relaciones entre las variables del modelo. También se encontró que la efectividad depende de una combinación de estrategias de enseñanza que incorporan los modos tecnológicos más interactivos de instrucción.

Se propone que con estos hallazgos se pueda continuar investigaciones documentales, puesto que sustentan el futuro desarrollo de los contenidos de cursos en línea, de tal forma que los instructores-asesores universitarios asuman un compromiso con la tecnología.

Por lo tanto, este último apartado presenta las conclusiones de la investigación. Para tal efecto se precisan los siguientes aspectos:

a. El trabajo que aquí se reporta, implico investigar cuatro teorías mediante la consulta de una muestra de artículos científicos para explicar la asociación de sus principales constructos y variables.

b. Al igual que Byrne (2010) se es coincidente en la fortaleza de la teoría estructural. En este sentido, la ventaja de la teoría representada en forma causal en el proceso, permite generar observaciones de múltiples variables. En otras palabras, las variables surgidas de los constructos, al poderlas incluir en el modelo, representan una alternativa de análisis para quienes investigan actualmente el ambiente virtual de aprendizaje, así como de la evaluación de sus implicaciones teóricas en el futuro.

c. El Modelo de ecuaciones estructurales y de acuerdo a su fundamentación teórica, permite confirmar el aspecto causal de los constructos.

d. La investigación se enfocó a resaltar las asociaciones entre los constructos que con mayor frecuencia se identificaron en los modelos estructurales. Las relaciones identificadas a través del modelo estructural entre las variables, permite identificar una relación de causalidad. Esto sin duda es de gran valor para continuarse estudiando en otras investigaciones.

Con esto último se concluye el informe de la investigación.

Presentación del artículo: 6 de agosto de 2015

Fecha de aprobación: 15 de abril de 2016

Fecha de publicación: 30 de abril de 2016

Ramírez-Carbajal, A. A. (2016). Constructos y variables del ambiente virtual de
aprendizaje, desde la perspectiva del modelo de ecuaciones estructurales. RED.
Revista de Educación a Distancia. 49(2). Consultado el (dd/mm/aaaa) en
http://www.um.es/ead/red/49

Constructos y variables del ambiente virtual de aprendizaje, desde la perspectiva del modelo de ecuaciones estructurales. Alfredo Ángel Ramírez-Carbajal.

Página 20 de 25 


\section{Referencias}

Abdalla, I. (2007). "Evaluating effectiveness of e-blackboard system using TAM framework: A structural analysis approach”, en AACE Journal, vol. 3, núm. 15, pp. 279-287.

Arbaugh, J. (2000). "Virtual classroom characteristics and student satisfaction with internet-based MBA courses", en Journal of Management Education, vol. 1, núm. 24, pp. 32-54.

Arbaugh, J. (2002). "Managing the on-line classroom: A study of technological and behavioral characteristics of web-based MBA courses", en Journal of High Technology Management Research, núm. 13, pp. 203-223.

Basabe, F. (2007). Educación a distancia en el Nivel Superior, Trillas, México.

Batista, J. y G. Coenders (2000). Modelos de ecuaciones estructurales, editorial la Muralla, Madrid.

Brown, T. (2006). Confirmatory factor Analysis for applied research, The Guilford Pres, New York.

Bunge, M. (2000). La investigación científica: su estrategia y su filosofía. Siglo veintiuno editores. México.

Byrne, B. (2010). Structural equations modeling with AMOS. Basic concepts, applications, and programming, Routledge, New York.

Chao-Min, Ch., Meng-Hsiang H., Szu-Yuan S., Tung-Ching L. y S. Pei-Chen (2005). "Usability, quality value and e-learning continuance decisions", en Computers \&Education, vol. 45, núm. 4, pp. 399-416.

Cho, V., Cheng, E., y J. Lai (2009). "The role of perceived user-interface design in continued usage intention of self-paced e-learning tools", en Computers \& Education, vol. 53, núm. 2, pp. 216-227.

Chuttur, M. (2009). "Overview of the technology acceptance model: origins, developments and future directions", en Sprouts: working papers on information systems, vol. 9. Núm. 37, pp. 1-21.

Coenders, G., Batista, J. y W. Saris (2005). Temas avanzados en modelos de ecuaciones estructurales, editorial la Muralla, Madrid.

Davis, F. (1985). A technology acceptance model for empirically testing new end-user information system: theory and results. Doctoral dissertation. Massachusetts Institute of Technology, Sloan School of Management, Cambridge, MA. 
DeLone, W. y E. McLean (2003). "The DeLone and McLean model of information systems success: a Ten-Year update", en Journal of Management Information Systems, vol. 4, núm. 19, pp. 9-30.

DeLone, W. y E. McLean (1992). "Information system success: the quest for the dependent variable", en Information System Research, vol. 1, núm. 3, pp. 60-63.

Fernández, R. (2009). Factores antecedentes en el uso de entornos virtuales de formación y su efecto sobre el desempeño docente. Tesis de Doctorado. España, Universidad Politécnica de Valencia, Facultad de Informática, Departamento de Organización de Empresas-Valencia.

Fishbein, M. y I. Ajzen (1975). Belief, attitude, intention and behavior: an introduction to theory and research. Reading, MA, Addison-Wesley: Technology Acceptance Model and E-learning.

Fong Ling, F.; Hung-Gi, Ch. y Y. Sheng-Chin (2007). "Activate interaction relationships between students acceptance behavior and e-learning", en APWeb/WAIM'07 Proceedings of the joint 9th Asia-Pacific web and 8th international conference on web-age information management conference on Advances in data and web management, vol. 4505, pp. 670-677.

Goon L.; Noornina, D.; Ramayah, T.; Noorliza K. y H. Hasmi (2005). "Impact of interface characteristics on digital libraries usage", en Malaysian Online Journal of Instructional Technology, vol. 2, núm.1, pp. 1-9.

Hernández, G. (2004). Paradigmas en Psicología de la Educación, Editorial Paidós Mexicana, México.

Holsapple, C. y A. Lee-Post (2006). "Desfining, assessing, and promoting e-learning success: an information system perspective", en Decision Sciences Journal of Innovative Education, January, vol. 4, núm. 1, pp. 67-85.

Hong, W.; Thong, J.; Wai-Man, W. y T. Kar-Yan (2002). "Determinants of user acceptance of digital libraries: an empirical examination of individual differences and system characteristics", en Journal of Management Information Systems, vol. 18, núm. 3, pp. 97-124.

Hoyle, R. (2012). Handbook of Structural Equation Modeling. The Guilford Press, New York.

Hsiu-Fen, L. (2007). "Measuring Online Learning Systems Success: applying the updated DeLone and McLean Model" en CyberPsychology \& Behavior, December, vol. 10, núm. 6 , pp. 817-820.

Jen-Her, W., Tzyh-Lih, H., Yi-Wen, L. y R. Tennyson (2008). "What determinates student learning satisfaction in a blended e-learning system environment? en Pacific Asia Conference on Information Systems, pp. 1-14.

Constructos y variables del ambiente virtual de aprendizaje, desde la perspectiva del modelo de ecuaciones estructurales. Alfredo Ángel Ramírez-Carbajal. 
Johnson, R.; Gueutal, H. y C. Falbe (2009). "Technology, trainees, metacognitive activity and e-learning effectiveness", en Journal of Managerial Psychology, vol. 24, núm. 6, pp. 545-566.

Joo, Y.; Lim, K. y S. Kim (2012). "A Model for Predicting Learning Flow and Achievement in Corporate e-learning", en Educational Technology y Society, vol. 15, núm.1, pp. 313-325.

Jung-Wan, L. (2010). "Online support service quality, online learning acceptance, and student satisfaction", en Internet and Higher Education, august, vol. 13, núm. 4, pp. 277-283.

Kaplan, D. (2009). Structural equation modeling: Fundations and extensions, 2a. Ed. U.S.A. SAGE Publications, Inc.

Martínez, F. y M. Prendes (coords.) (2007). Nuevas Tecnologías y Educación, Pearson Educación, Madrid.

Martínez-Otero, V. y L. Torres (2005). “Análisis de los hábitos de estudio en una muestra de alumnos universitarios" en Revista Iberoamericana de Educación, RIE, vol. 35, núm. 7, pp. 1-8.

Martínez-Torres, M.; Toral, S.; Barrero, F.; Gallardo, S.; Arias, M. y T. Torres (2008). "A technological acceptance of e-learning tools used in practical and laboratory teaching, according to the European higher education area", en Behaviour \& Information Technology, November-december, vol. 27, núm. 6, pp. 495-505.

Martins, L. y W. Kellermans (2004). "A Model of Business School Students' Acceptance of a Web-Based Course Management System", en Academy of Management Learning and Education, March, vol. 3, núm. 1, pp. 7-26.

Mueller, D. y S. Strohmeier (2011). "Design characteristics of virtual learning environments: an expert study", en Computers \& Education, vol. 57, núm. 4, pp. 2505-2516.

Mueller, D. y V. Zimmermann (2009). "A learner-centered design, implementation, y evaluation approach of learning environments to foster acceptance", en International Journal of Advanced Corporate Learning, vol. 2, núm. 3, pp. 50-57.

Nov, O. y C. Ye (2008). "Users' personality and perceived ease of use of digital libraries: The case for resistance to change", en Journal of the America Society for Information Science and Technology, vol. 59, núm. 5, pp. 845-851.

Panadero, E. y Alonso-Tapia, J. (2014). Teorías de autorregulación educativa: una comparación y reflexión teórica. Psicología Educativa, Vol. 20. Núm. 1, p. 11-22.

Parcerisa, A. (2007). "Los materiales, recurso para el aprendizaje", en Aula de innovación educativa, núm.165, octubre, pp. 7-11.

Constructos y variables del ambiente virtual de aprendizaje, desde la perspectiva del modelo de ecuaciones estructurales. Alfredo Ángel Ramírez-Carbajal. 
Pei-Chen, S.; Tsai, R.; Finger, G.; Yueh-Yang, Ch. y D. Yeh (2008). "What drives a successful e-learning? An empirical investigation of the critical factors influencing learner satisfaction", en Computers \& Education, núm. 50, pp. 1183-1202.

Picciano, A. (2002). "Beyond student perceptions: issues of interaction, presence, and performance in an online course", en Professor, School of Education, July, vol. 6, núm. 1, pp. 21-37.

Pituch, K. y L. Yao-Kuei (2006). "The influence of system characteristics on $e$-learning use”, en Computers \& Education, núm. 47, pp. 222-244.

Po-An, H. y Cho, V. (2011). "Comparing e-learning tools' success: The case of instructor-student interactive vs. self-paced tools", en Computers \& Education, vol. 57, núm. 3, pp. 2025-2038.

Poelmans, S.; Wessa, P.; Milis, K.; Bloemen, E. y C. Doom (2008). "Usability and acceptance of e-learning in statistics education, based on the compendium plataform", en Proceedings of the International Conference of Education, Research and Innovation, pp. 1-10.

Roca, J.; Chao-Min, Ch. y F. Martinez (2006). "Understanding e-learning continuance intention: an extension of the technology acceptance model", en International journal of human computers studies, núm. 64, pp. 683-696.

Ruiz, M.; Pardo, A. y R. San Martín (2010). “Modelos de ecuaciones estructurales”, en Papeles del Psicólogo, Sección Monográfica, vol. 31, núm. 1, pp. 34-45.

Sahin, I. y M. Shelley (2008). "Considering students perceptions: the distance education student satisfaction model”, en Educational Technology \& Society, vol. 11. Núm. 3, pp. 216-223.

Schmelkes, C. (2007). Manual para la presentación de anteproyectos e informes de investigación (tesis). $2^{\text {a }}$ edición, México, Oxford.

Seddon, P. (1997). "A respecification and extension of the delone and mclean model of is success", en Information systems research, vol. 8, núm.3, pp. 240-253.

Selim, H. (2007). "Critical success factors for e-learning acceptance: Confirmatory factor models", en Computers \& Education, april-september, núm. 49, pp. 396-413.

Shu-Sheng, L. y H. Hsiu-Mei (2003). "An investigation of user attitudes toward search engines as an information retrieval tool", en Computers in Human Behaviors, núm. 19, pp. 751-765.

Shu-Sheng, L.; Weng-Cheng, Ch.; Wu-Hsiung, H. y H. Hsiu-Mei (2006). "Attitudes toward search engines as a learning assisted tool: Approach of a Liaw and Huang's research model", en Computers in Human Behavior, March, vol. 22, núm. 2, pp. 177 190.

Constructos y variables del ambiente virtual de aprendizaje, desde la perspectiva del modelo de ecuaciones estructurales. Alfredo Ángel Ramírez-Carbajal. 
Silva, C. y I. Schiattino (2008). “Modelos de ecuaciones estructurales ¿qué es eso?”, en Ciencia y Trabajo, julio-septiembre, vol.10, núm.29, pp. 106-110.

Stahl, G.; Koschmann, T. y D. Suthers (2006). "Computer-supported collaborative learning: an historical perspective", en R. K. Sawyer (Ed.). Cambridge handbook of the learning sciences. Cambridge, UK: Cambridge University Press. pp. 409-426.

Su-Chao, Ch. y T. Feng-Cheng (2008). "An empirical investigation of students' behavioral intentions to use the online learning course websites", en British Journal of Educational Technology, vol. 39, núm. 1, pp. 71-83.

Su-Houn, L.; Hsiu-Li, L. y P. Cheng-Jun (2005). "Applying the technology acceptance model and flow theory to online e-learning users acceptance behavior", en Issues in Information Systems, vol. 6, núm. 2, pp. 175-181.

Thong, J.; Hong, W. y T. Kar-Yan (2002). "Understanding user acceptance of digital libraries: What are the roles of interface characteristics, organizational context, and individual differences?" en International Journal of Human-Computer Studies, vol. 3, núm. 57, pp. 215-242.

Tobing, V.; Hamzah, M.; Sura, S. y H. Amin (2008). "Assessing the acceptability of adaptive e-learning system", en Proceedings of the 5th International Conference on eLearning for Knowledge-Based Society, pp. 1-10.

Wen-Shan, L. y W. Chun-Hsien (2011). "Antecedences to continued intentions of adopting e-learning system in blended learning instruction: a contingency framework based on models of information system success and task-technology fit", en Computers \& Education, vol. 58, núm. 1, pp. 88-99.

Ya-Ching, L. (2006). "An empirical investigation into factors influencing the adoptions of an e- learning system", en Online Information Review, vol. 30, núm. 5, pp. 517541.

Yeung, P. y E. Jordan (2007). "The continued usage of business e-learning courses in Hong Kong corporations", en Education and Information Technologies, vol. 12, núm. 3, pp. 175-188.

Yi-Shun, W. (2003). "Assessment of learner satisfaction with asynchronous electronic learning systems”, en Information \& Management, núm. 41, pp. 75-86.

Yi-Shun, W.; Hsiu-Yuan, W. y D. Shee (2007). "Measuring e-learning systems success in an organizational context: Scale development and validation", en Computers in Human Behavior, núm. 23, pp. 1792-1808.

Yurén, M. (2000). Formación y Puesta a Distancia, Paidós Mexicana, México.

Constructos y variables del ambiente virtual de aprendizaje, desde la perspectiva del modelo de ecuaciones estructurales. Alfredo Ángel Ramírez-Carbajal. 